\title{
Geophysical field disturbances and quantum mechanics
}

\author{
Vladimir Kuznetsov ${ }^{1, \star}$ \\ ${ }^{1}$ Institute of Cosmophysical Research and Radio Wave Propagation FEB RAS, Paratunka, Russia
}

\begin{abstract}
Quantum processes impact into physics of geophysical field disturbances is discussed here in examples of phenomena such as an earthquake with processes preceding and accompanying it, volcanoes eruptions and diamond exploding pipes. Physics of shock waves generation in ionosphere and atmosphere, mechanism of atmosphere phenomena in supercooled clouds recorded by a stormglass is considered. The report treats of physics of ball and dark lightning, of generating in atmosphere the high-energy particles involved in sprites occurrence, and so on.

Geophysical phenomena considered here have no clear and consistent interpretation in the context of classical physics. We attempt to involve the recent achievements of quantum physics namely the quantum entanglement between elementary particles implicated in considered phenomena.
\end{abstract}

\section{Introduction}

It is well known that geophysics describes nature phenomena occurring in solid Earth (earthquakes, volcano eruptions, heat flow), in oceans (tsunami), in atmosphere (clouds formation, climate, atmosphere electricity, thunderstorms, sprites). Geophysics is a complex of sciences, which explore the structure of the Earth by the physical methods. Each phenomenon implies the formation of "its own "fields unique to it, they are thermal field of the Earth, gravity field, the Earth's magnetic field and the electromagnetic field. The division into fields determines the division of geophysics into relevant disciplines, such as meteorology, oceanography, etc. Geophysics phenomena such as earthquakes, volcano eruptions, lightning and sprites, climatology, etc. appear not to fit the only one section. This is particularly noticeable when talking about possible methods of prediction. Let us cite, for example, some of the ways of earthquakes forecast. They are acoustic monitoring, and monitoring of electric and magnetic fields, changes of gravity and stresses in lithosphere, monitoring of heat flow, change in groundwater level, etc. As many of these fields are experiencing a perturbation on the eve of an earthquake they must be considered jointly. Sometimes it turns out that it is impossible to find an "agent" which would be manifested simultaneously in these fields. The fact is that physicists use to solve the problem in the frames of one method inside the so-called classical physics.

Currently the situation in science began to change radically. To explain some phenomena the physicists appeal to quantum mechanics assuming the so-called classical physics in which frames the phenomena have no apparent explanation to be a component of quantum mechanics. Here physicists has called attention to a particular matter state, named quantum entanglement (QE) (quantum entanglement, nonlocality). Quantum properties were considered to manifest themselves only at very low

\footnotetext{
${ }^{\star}$ Corresponding author: vvkuz38@mail.ru
} 
temperatures close to the absolute zero. Recently it was shown that it is not entirely true. Secondly, entangled substances have a property effecting as expressed by Einstein, a "supernormal long-range action". Otherwise, particles of substances in QE state behave like a single substance, despite the fact that there are no interaction between its parts and particles can be at any large distance from each other. In quantum mechanics it means that all linked particles of a substance are described by a single wave function and they have a specific coherence. It is not appropriate to object to this approach from the point of GTR. Thirdly, talking about the role of QE in nature it is worth to refer to the recently published paper stating that the essence of gravity consists in quarks and antiquarks QE [1]. The development of this approach seems to allow to solve the problem of gravity quantization.

Is QE common at the Earth? The answer to this question is given at a recently published article alleging that "everything is linked" in the world [2]. According to the authors QE originates from the creation of the Universe i.e. from the Big Bang. In fact, a typical particle is entangled with many particles far outside our horizon. However, as QE is evenly propagating by a volume the two randomly chosen particles are unlikely to be associated through reduced density matrix which applies to any pairs that are separable [2].

Here we will focus on the fast perturbations of the geophysical environment, called quakes. Quake is the environment shaking against the background of its initially calm conditions. Where and how do quakes occur? In the monograph [3] these phenomena refer to such ones (not including a well known earthquake) like: ice quake, wood quake, protein quake, etc. Author of this monograph rightly believes that in such cases a violation of the fluctuation-dissipative theorem has occurred. We refer lightning (ball and dark), sprites, etc. to quantum phenomena. Obviously, the broader is the range of quake, the more difficult is to select an environment with a universal QE. In the subsequent discussion we will restrict ourselves to QE of hydrogen bonds protons (HB).

Hydrogen bond - a special type of bonding due to the fact that the hydrogen atom associated with a strongly electronegative element (nitrogen, oxygen, fluorine, etc.) lacks of electrons and it therefore is able to interact with the lone electron pair of another electronegative atom of the same or another molecule (the total proton). The presence of $\mathrm{HB}$ and their cooperative properties, particularly in water, lead to the fact that water properties change depending on the number of HB. For example, in ice there is a lot of $\mathrm{HB}$ and their number is decreasing with ice melting. The number of HB small in melt is less in hot water and there is almost no HB in boiling water. If water completely has lost its ability to form $\mathrm{HB}$, it would turn into vapor, condensing into liquid at $100{ }^{\circ} \mathrm{C}$. Present not only in water but also in the lithosphere hydrogen bonds are determining its special properties. The mechanism of $\mathrm{HB}$ is that the electron density on the line $\mathrm{H} . . . \mathrm{O}(\mathrm{N}, \mathrm{F}$, etc.) is shifted to the electronegative atom generating a nearing of electrons of electronegative atoms in the neighboring molecules and the distance $\mathrm{O}-\mathrm{H}, \mathrm{O}$ $\mathrm{N}$, etc. decreases.

Data on QE. "A surprising feature of the quantum world is that one particle instantly affects "entangled" with it another one, no matter how far apart they are. The term "entanglement" to describe this phenomenon came up with Schrödinger who like Einstein was not a fan of what Einstein called a "supernormal long-range action". But despite their skepticism, the quantum "entanglement" proved in many experiments is one of the most fundamental ideas in quantum mechanics..." ([4], p. 235). Quoting the book of S. I. Doronin, "Quantum magic" [5]: "The matter, that is the matter and all known physical fields are not environment basis but represent only a small part of the total Quantum Reality."

Some basic definitions from quantum mechanics:

Decoherence is a physical process in which the locality breaks and quantum entanglement between the constituent parts of the system reduces in its interaction with the environment. Her the subsystems "manifest" from their nonlocal states as separate independent elements of reality, they separate from each other, acquiring visible local forms. 
Quantum tunneling is a quantum mechanical effect of particles passage through the energy barrier. Similar to the passage through the tunnel this process is called a tunneling. There is no analogue in classical mechanics.

Nonlocality is a feature of entangled states which cannot be related to the local elements of reality. It is not relevant to waves, fields and to the classical energy of any kind and type. Quantum nonlocality have no classical analog and it cannot be explained within classical physics.

Nonlocal correlations (quantum correlations) - a specific effect of nonseparability (quantum entanglement) which consists in concurrent behavior of separate parts of the system. It is a "telepathic" association of objects when each of them feels the other one "as itself." This "supernatural" coupling between distant objects is not explained by classical physics. Unlike regular interactions restricted for example by the light speed, the nonlocal correlations are instantaneous, that is, changes at each part of the system affects its other parts at the same the time regardless of the distance between them. Quantum physics has revealed the mechanism of this coupling, it got into a quantitative description of coupling laws and gradually begins to use in technical devices.

Nonseparability (quantum entanglement) - the inability to divide the system into separate and completely independent parts.

The principle of superposition of states - if the system can be in different states, it may be in a state which is a simultaneous "superposition" of two or more states from this set.

Recoherence - reverse to decoherence the process is regenerating a quantum entanglement between the system components.

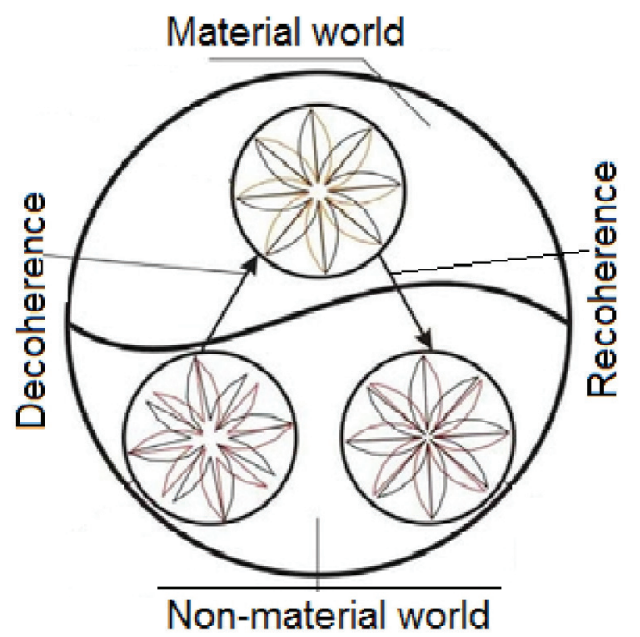

Fig. 1. Quantum jumps on the border between the material world and the non-material one [6]

\section{Physics of the earthquake}

As an unsolved problem physics of the earthquake challenges humanity for over 100 years and still remains unclear. As was recently announced by experts of the popular journal LiveScience, one of the ten mysteries of the Universe is formulated approximately as: "What is happening in the "heart" of the earthquake?" 
A specialist in quantum mechanics Professor Jim al-Khalili formulates the 10 tasks of modern physics in his book "Paradox. Nine great mysteries of physics" [7]. Among them, the second position is for dark matter, the third one for dark energy, and question "will we learn one day to predict earthquakes?" is at the ninth position. To answer this question I stated about 25 years ago: "already begun the earthquake does not know what happens further." In other words there is no hope for reliable prediction. Intuitively I tried to find quantum "roots" of an earthquake and then I confirmed my statement.

Here the purpose is an attempt to understand physics. In previous papers the author showed that the earthquake can be presented as the output of a shock wave to the Earth surface, causing the emergence of the so-called strong motions of ground [8]. Let's follow this conception on data of quantum mechanics.

\section{Experiments data}

Our model is based on fundamentally new approaches to the explanation of the results obtained in research of rock samples under their compression by powerful presses. The authors have been repeatedly recorded spontaneous amplification of the intensity of acoustic emission followed by its spontaneous determination [8-10]. The author [8] attempted to explain this phenomenon from the point of view of self-organization in coherent structures by interaction of sound waves with opening cracks. The effects of acoustic emissions amplification was suggested to be similar to optical superradiance of Dicke [11].

The effects of acoustic superradiance are noted to occur not in all types of rocks. For example, the acoustic emission of granites (granodiorites) of two different types: Oshima (fine-grained) and Inada (coarse-grained) under their loading was revealed to be different though the samples had a formal resemblance [9]. Whereas samples of granites from Oshima deposit showed a sharp increase in intensity of acoustic emission, there was no effect at samples of granites from Inada. This result repeatedly confirmed suggests that Earth interior there may be geological bodies which rheology enables self-organization and generating of an earthquake inside them, while in other bodies at first glance identical to the first ones such phenomena do not occur.

The results of laboratory experiments and field observations (Fig. 2A) show that constant acoustic background has a fluctuating character $(\sim \sqrt{N})$, where $N$ is a complete, the maximum possible number of sound impulses arriving at the receiver from the loaded sample. If impulses are generated coherently $\mathrm{N}$ appears. For comparison (Fig. $2 B$ ) shows the result of the experiment on the effect of quantum entanglement. Pay attention to the fact that in these figures the form of signals is identical. In our model we accept that the acoustic impulses are generated due to changes of hydrogen bonds length (breaks) at the rock samples.

\section{The formation of SW at the lithosphere}

Let's discuss the experiments on high-pressured kalicinite (KHCO3), the mineral in which hydrogen bonds show the capacity for quantum entanglement. On compression the structure of hydrogen bonds changes simultaneously over the whole volume of the sample (Fig. 3) resulting in the change of its unit-cell volume (or density) and correspondingly (according to our model) in the SW generation.

Similar results were obtained for other minerals containing hydrogen and oxygen (nitrogen, fluorine). By data of many authors such structural phase transition of hydrogen bonds happens instantly over a large volume and this phenomenon is called a cooperativity.

The parts of our model: 1) a shock wave formation at the breakup of the medium characteristics; 2) evaluation of an earthquake energy; 3) output of the shock wave at the surface and physics of 


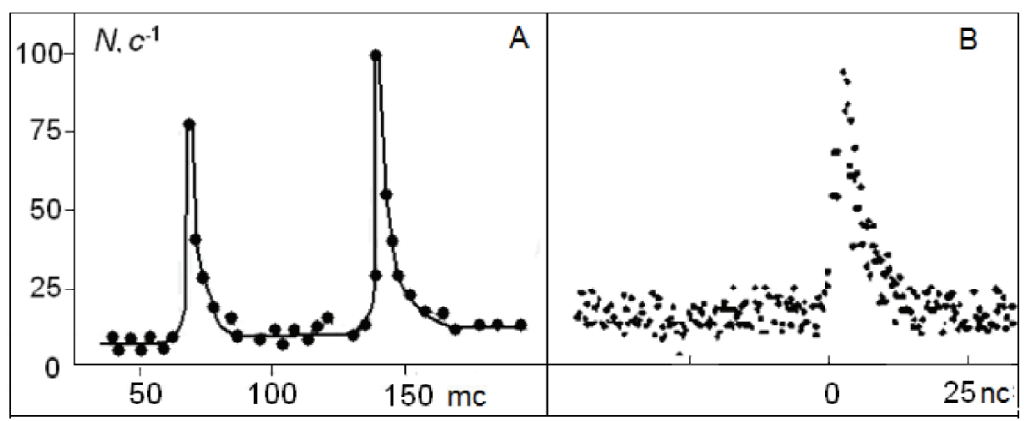

Fig. 2. A) Formation rate of sound impulses in diabase under a constant uniaxial compressive stress [10]. B) Number of photons pairs registered as a function of delay between the moments of two photons detection. Flat plot corresponds to random coincidences between uncorrelated photons, the peak corresponds to quantum entanglement [12]

accompanying phenomena; 4) model of an earthquake source - are discussed in [13]. As these parts are not associated with quantum mechanics as well as with material presented further they are not treated here. Model of aftershocks as quantum phenomena follows.

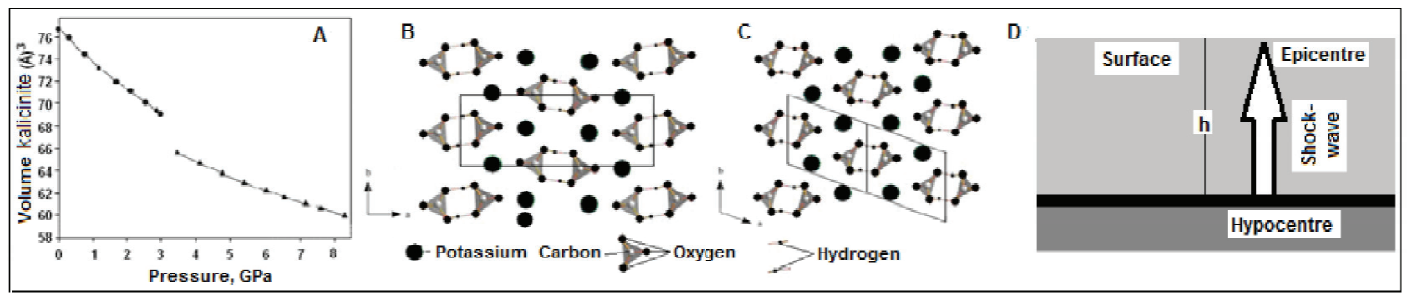

Fig. 3. The change of the unit-cell volume $(\Delta V=V=4 \%)$ for kalicinite at the pressure of $3.2 \mathrm{GPa}$ - $A$, the change of kalicinite crystal structure - $B, C$ [12]. Shock wave generation inside the layer of lithosphere containing hydrogen bonds $-D[13]$

\section{Aftershocks}

After a strong earthquake at the region of its source a swarm of recurrent earthquakes with the depth of their hypocenters as a rule higher than the plane of the main shock occur. After the main shock the decline of aftershocks number in time is defined by a hyperbolic law (Omori law $N(t) \sim 1 / t \zeta$, where $\zeta>1$ ). As usually aftershocks fill the space previously occupied by the source of the main shock its volume may be evaluated in such a way (Fig. 4-C). Almost all hypocenters of aftershocks, some of them are comparable in magnitude to the main shock, are located above its hypocenter.

Though after a deep earthquake aftershocks are virtually absent, however, this relation is known to be rarely violated. After the deep $(564 \mathrm{~km})$ earthquake of 9 March 1994 (with a magnitude of $M_{W}=7.6$ ) near the Tonga the authors [14] observed the first series of aftershocks using eight broadband seismographs. Aftershocks ( 82 events) have been fading after the main shock for 42 hours according to the power law with magnitudes from 3.8 to 6.0. The main shock and most of aftershocks 
were located along the vertical plane of the main shock with the maximum distance from it of not more than $5 \mathrm{~km}$.

Seismically active medium including entangled elements has a number of unique properties among which we are interested in the ability to memory. To use this feature, physicists are trying to create a quantum computer. In seismology this property manifests itself when, along with the main earthquake, foreshocks and aftershocks occur. If medium have not such a property, it is not able to generate foreshocks and aftershocks.

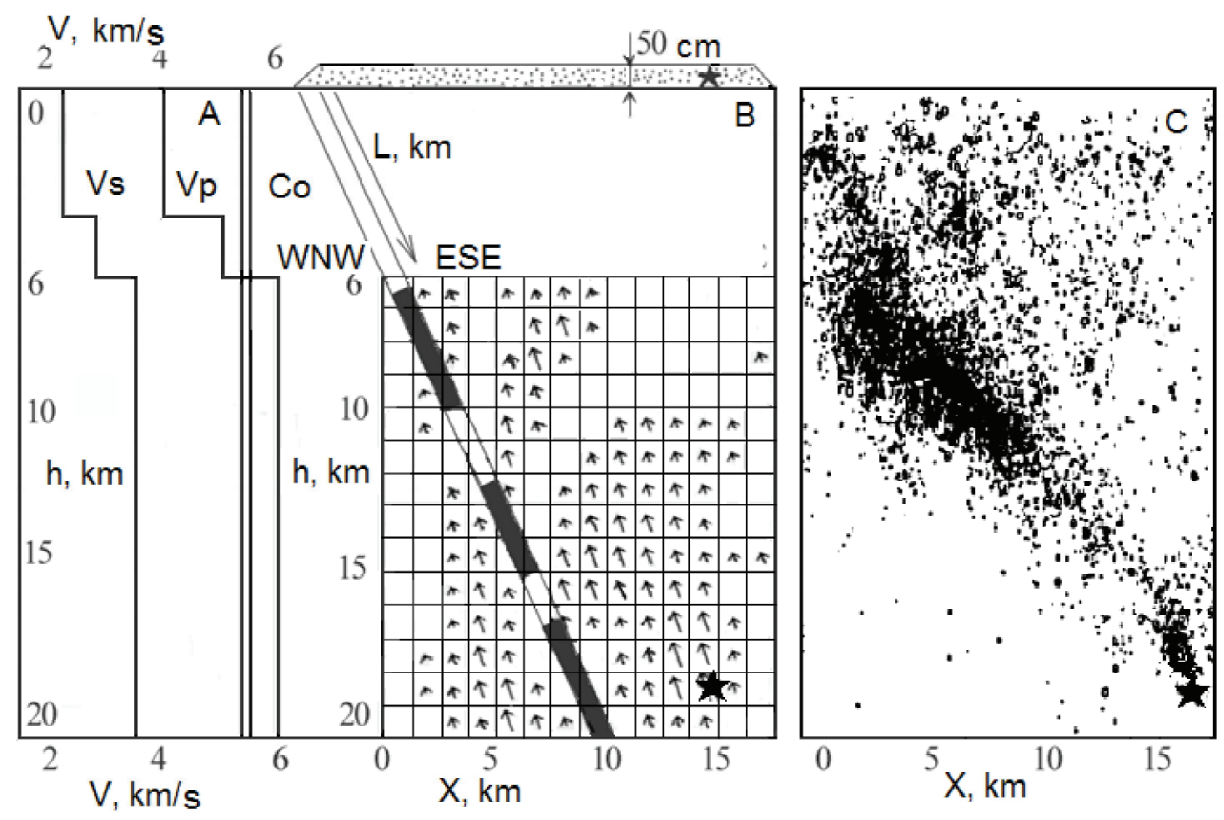

Fig. 4. Model of the Northridge earthquake ruptures (center) [14]. Left - depth distribution of $P-$, $S$-waves and shock waves velocities. Right - aftershocks [14]

\section{Quantum effects in physics of earthquakes}

Let's refer to Fig. 2, which shows that the rock sample loaded by press emits a sound impulse. It is generally accepted that the cause of these impulses is the cracks formation. We will not criticize this model [15] here. Here we suppose another mechanism of acoustic emission. Assuming that the rock sample includes HB as it follows from Fig. 3-B and 3- $C$ we consider a model of acoustic emission generation due to the motion of a proton in the sun.

Let's use the model of HB [4], where the proton in the hydrogen bond linking the two pairs of DNA bases is regarded as being on two springs such that it can oscillate from side to side. It has two possible stable positions modeled here as a double energy well. The left-hand well (corresponding to the unmutated position) is slightly deeper than the right-hand well (the tautomeric position), and so the proton prefers to sit in the left one.

In Fig. 5 shows the component of the system [4]. Remember, in quantum mechanics a proton is an elementary particle which is simultaneously a wave. The movement of proton will be recorded as a phonon, i.e., the sound impulse. Moving along the hydrogen bond the proton meets an energy 
barrier and passes through it due to the effect of quantum tunneling. It was Per-Olof Lundin, Swedish physicist, who suggested that the protons in hydrogen bonds move by quantum tunneling resulting in formation of mutational forms of the nucleotides ([4] p. 277).

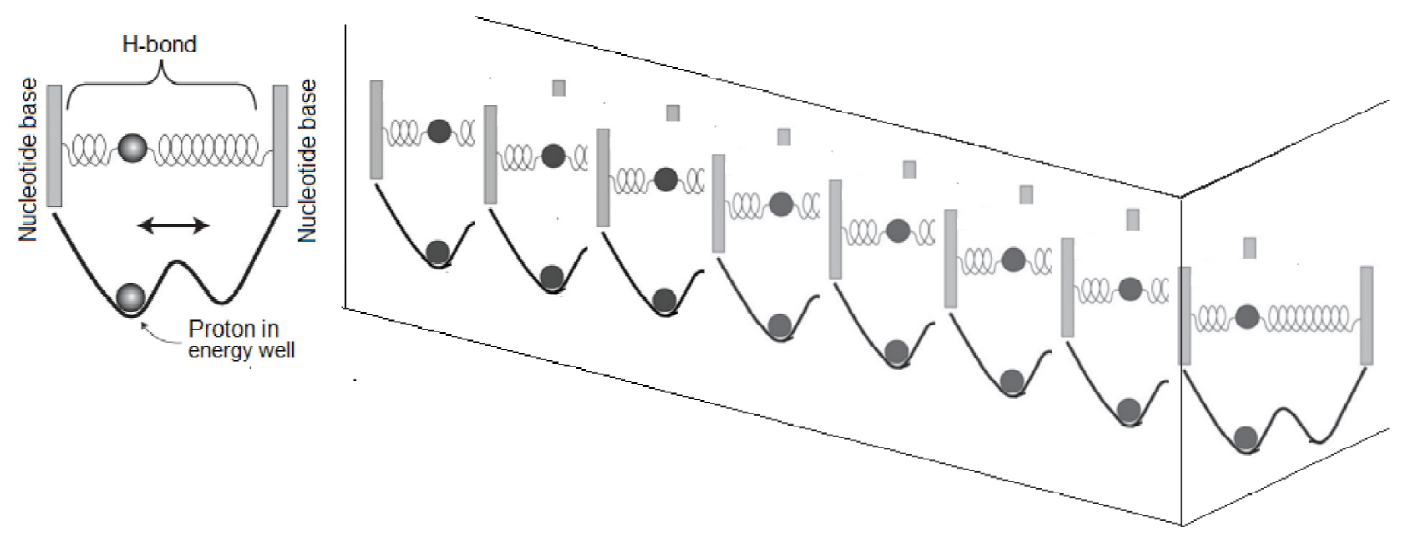

Fig. 5. Proton in hydrogen bonds linking the two pairs of DNA bases [4]. The range of $\mathrm{HB}-$ an element of an earthquake source

Why there is a sharp increase in the number of sound impulses, which is then reduced to the previous background values, and soon the process is repeated. In quantum mechanics the background acoustic emission is a random process of proton moving when protons become coherent. Upon reaching full coherence by protons of $\mathrm{HB}$ in a sample volume decoherence occurs (Fig. 1). The consequence of decoherence is a rapid increase in the number of sound inpulses $\mathrm{N}$ followed by recoherence, the system enters quantum domain. This stage is called a virtual quantum entanglement, here HB protons again "find" the same protons in the same quantum states. The process repeats.

Fig. 2- $B$ shows the result of laboratory experiments on quantum entanglement of correlated photons [12]. Decoherence similar to that treated above is recorded: turning into classical domain the system turns back into quantum one in the process of recoherence.

In our model the reason for changing of $\mathrm{HB}$ potential is the pressure size of the press in laboratory experiments and the magnitude of gravity in nature. In [16] the dependence of rate constant for tunnel solid-phase reactions on temperature and pressure is considered. The temperature dependence is determined by intermolecular and weak intramolecular vibrations in reagents, leading to oscillations in the permeability of the potential barrier for the tunneling atom. The external pressure accounts for static reduction in the width and height of the potential barrier (dashed line in Fig. 5) under hydrostatic compression of matrix that leads to a higher transparency of the barrier.

Synchronous protons jump from top energy level down to the lower one as decoherence leads to coherent addition of sound impulses and shock wave (SW) initiation. Propagating from the hypocentre up to epicentre SW carries a pressure additional to lithostatic one at its front. This pressure, in turn, causes the same HB protons jump in upper layers of an earthquake source and an aftershock generation. An obvious condition for aftershock occurrence involves quantum entangling of protons over the total volume. If such entanglement occurs not only within the earthquake source, but also with HB protons of the external environment then SW can occur for these protons. As will be shown below, the aftershock can be generated between the protons of the lithosphere and supercooled water clusters of the cloud. It follows that decoherence in a quantum system is an earthquake. 


\section{The shock wave initiator and source of geophysical phenomena}

After I made sure that the SW output to the earth surface very accurately correspond to the observed phenomena, named in seismology by "strong motions", I tried to test whether SW is a quake and whether it is possible to use this idea in other geophysics problems? It turned out that there are such problems and their solutions are given below in a brief overview. Their full discussion is presented in my articles and reports.

\section{$8 \mathrm{SW}$ and volcano formation}

My multiyear analysis of volcanological literature allows to make a few important observations: first, I noticed that volcanoes are in many seismically active regions and they are often close to epicenters of earthquakes. Second, the seismic pattern shows that beneath every volcano there is a magma chamber, and a volcano is a cone. Third, this chamber is not connected by a transport path of lava with another volume of the melt larger than the first one. Fourth, source of heat required for local melting of basaltic layer or mantle is an unsolved problem of volcanic activity manifestation. This melting should be localized, since a passage of seismic waves indicates that the crust and the upper mantle are solid. Moreover, thermal energy should be sufficient for melting of huge volumes of solid material. I could not come up with a source of heat, and sources mentioned in articles didn't suit. My idea is in the SW which, coming at the surface, produces strong ground motions accompanying earthquake and if medium is fractured then SW is absorbed by this environment, that is a local source of heating. There are well-known data on medium heating by SW. For example, [18] large-scale molecular modeling of SW propagation dynamics and quasi-isoentropic (adiabatic) compression of defective crystals have shown that so-called virtual melting is possible in distribution of SW over plastic environment. This effect is provided by reducing of melting point of the substance on $80 \%$, for example, for copper $\Delta T \approx 4000 K$, at the SW front. SW interacting with plastic environment, results in supercooled liquid.

This idea, if it appears correct, allows to explain why magmatic chambers do not cool for a long time being heat sources and they are used, for example, at thermal stations, etc. It would be possible to assess, for example, how many earthquakes (or SW) in the area of the volcanic source are necessary to provide heating of underground thermal source.

Volcano is usually conical and pouring out of its peak magma forms this cone. Compare a normal volcano with the structure of the volcanoes, for example, that on Io, Jupiter's moon, or with the famous supervolcano Yellowstone. Supervolcanoes have no cones and they are similar to plumes with magma pouring out of the outer core of the Earth by a channel [19].

\section{SW and the formation of blow holes}

Physical mechanism of diamonds elevating to the Earth surface is considered to be realized by forming blow holes or diatremes which resemble volcanoes, as diamonds are formed here at pT-conditions typical of the depths of about $100 \mathrm{~km}$. How to transport a diamond with this depth? To this question the accepted answer by far is the volcano. Conception of diamonds elevating by volcano eruptions meets contradictions. A radically new idea of diamonds creating at small depths with their further deposition at the Earth surface is based on the model of the Hot Expanding Earth being developed by the author [20], his new physics of earthquakes, based on the SW formation in the lithosphere, its output at the surface and its interaction with a rarefaction wave initiated here is proposed. Model of Hot Earth accounts for changing of pT-parameters at the Earth's surface in its evolution, specifically, increasing of the Earth radius and, accordingly, reducing of pressure and temperature gradients [21]. 
Diamonds turned out to be formed about a milliard years ago at depth of $5-10 \mathrm{~km}$ and then they were delivered close to the earth surface through the environment moving after SW which acted as diatreme.

\section{$10 \mathrm{SW}$ in ionosphere}

New model of the formation of shock acoustic waves (SAW) in the atmosphere and the ionosphere is discussed [13].

Recording propagation of the shock acoustic waves (SAW) in ionosphere using a wide network of GPS stations modern research methods of phenomena in atmosphere and ionosphere don't solve the problem of mechanism of SAW generation. We consider attempts to assign occurrence of SAW to strong ground motions during an earthquake being incorrect, because the speed of these motions for SW initiation is too low.

Model of SAW generation as an aftershock of lithosphere earthquake is proposed here. Quantum entanglement in "earthquake source - supercooled water clouds" system postulated in our model may occur on HB protons in the source substance and, of course, on these present in the aquatic environment of such clouds. Our model removes all time and space inconsistencies observed between the earthquake source and SAW hypocenter. The same postulate is applied to aftershocks.

On the one hand the occurrence of SW in a cloud of supercooled water leads to the generation of a "hole" in the cloud, and on the other to the high-speed release of ice piece from clouds. It is a product of activitiy of rarefaction wave, which occurs simultaneously with SW. Probably, this mechanism leads to the appearance of UFOs and, at the same time, if ice speed is higher than that of sound in air there is a so-called "bombshell".

Not that various mechanisms of entanglement (many-particle quantum entanglement) except those adopted here (for simplicity) for HB protons are discussed in [22], but they will complicate our task greatly.

\section{Shock-wave model of explosive hole}

Model of explosive hole formation on the Yamal Peninsula and release of gas hydrates on Bennett island is suggested [23]. The reason for the such phenomena is the SW impact on the volume of gas hydrates deposits at the permafrost. Gas hydrates are destroyed through SW passing as an earthquake or an icequake which frequently occur in of gas hydrates deposits. Explosive hole formation or release of gas hydrates on Bennett island should need a series of earthquakes (shock waves).

\section{Rock bump - SW output at a mine}

Model in which a rock bump (an induced earthquake) as SW output at the inner wall a mine is discussed [24]. An associated rarefaction or stretching wave is moving in reverse (into the wall) direction. Ground motions in the direction of SW propagation with a velocity much smaller than that of SW occur. Phenomena on the inner surface of a mine result in the release of coal dust and methane. Spatial separation of charged particles causes an electrical breakdown, which initiates methane explosion.

\section{Storm glass monitoring of quantum entanglement}

English hydrographer admiral Robert Fitzroy was the first who described a strange barometer called then Fitzroy storm glass (SG) [25]. In 19-th century he used it for weather prediction namely for 
storms forecasting. Camphora crystallization within the liquid was believed to be related to the weather. Crystals form, height and amount were interpreted.

Numerous researchers of SG insist that SG has a little value in weather prediction and its operation principle is unclear. We were solid with these conclusions before we arrive at Kamchatka where the device began to record cyclones far before their coming.

Returning to Novosibirsk we made three similar SG. They turn to describe cyclones behavior at Kara Sea rather than Novosibirsk weather. Interested in quantum effects I develop now a quantum model of SG. As I propose SG quantum entangles with supercooled clouds of north cyclones rather than south cyclones. We called this device by an entanglemeter suggesting that it records a quantum entangling degree between clod supercooled water and camphora with its hydrogen bonds. Protons shifting in HB accounts for crystal growth at SG.

\section{Lightning, MeV-particle fluxes, sprites}

Ball lightning (BL) is unique a natural phenomenon [26]. First, this phenomenon is far ahead of all ones in the number publications. Second, despite a huge research number, it can not interpreted in the frames of classical physics. The only way to understand the BL nature we see is to consider its physics from quantum mechanics principles and achievements.

Here we propose a model in which the properties of SHM follow from the context of nonlocal multiparticle quantum entanglement (entanglements, the coherence - entanglement) between protons of hydrogen bonds (HB) in water clusters after the destruction of dark lightning. Dark zip. Discusses the problem of emergence in the clouds streams of high-energy particles. Describes several situations in which such discovered streams of dark lightning, preceding the ordinary and create additional ionisation, streams of fast electrons Mawei energy prior to the earthquake, lightning strikes, triggering the appearance of ball lightning, light effects, sprites, etc. All these phenomena seem to have a common origin based on the manifestation of the effects of quantum coherence of protons in hydrogen bonds of water clusters in the cloud [27].

\section{Dark lightning}

Emergence of high-energy particle fluxes in clouds is discussed here. Several cases in which they were discovered are dark lightning, preceding an ordinary one and creating additional ionisation, prior to an earthquake fluxes of fast electrons with $\mathrm{MeV}$-energy, lightning strikes, triggering an appearance of ball lightning, light effects, sprites, etc. All phenomena listed seem to have a common origin based on manifestation of quantum coherence of HB protons in water clusters of a cloud [27]. Discovered as a phenomenon in 1991 dark lightning (DL) is a flux of fast electrons, moving with velocities close to that of light that sharply distinguishes them from ordinary lightning, which electrons are not so rapid. Ultrafast electrons of DL colliding with the air molecules give rise to gamma rays that generate electrons and their antiparticles, whose subsequent annihilation with air produces more gamma rays. Lightning familiar to us follows the track left by DL which electric current is less by several orders of magnitude than that of usual lighning. DM voltage is known to be much higher than the potential of a storm cloud.

\section{Quantum effects in atmosphere electric field}

"Crystal structure", similar to that which occurs in crystalline dusty plasma is a possible reason for the stability of water-jumper (bridge) between two water containers under the influence of a constant 
electric intensity. Perhaps a similar mechanism is responsible for tornado water column behavior like that of a rigid body [28]. An interesting experiment with a stable water bridge between two beakers containing water and platinum electrodes was described in 2007 (referenced in [28]). Each electrode had a constant voltage of $15-20 \mathrm{kV}$. Touching from the start the beakers were pulled apart after the voltage being applied and water in both beakers rose by contacting beakers walls up to connecting of both water flows moving towards each other. Water bridge ("bridge") of circular section with a diameter of 2-4 $\mathrm{mm}$ and a length of about 2-3 $\mathrm{cm}$ was observed.

In the 80-90 I was developing my hot model of the expanding Earth. At that time I was interested in research on so-called "plasma crystal" and its quantum properties. Analyzing the results of this research, I created my model of the Earth inner core [20]. The idea of a "plasma crystal" helped me find the most likely model of water bridge: under the electric field water of bridge behaved as a rigid body.

It is known that water column of tornado behaves as a rigid body. If we imagine that water column of tornado is a water bridge with a potential of a parent cloud the problem would be solved. I have not found here quantum entangled HB protons but it is revocable.

\section{Conclusions}

In February 2013 I put sited my Memorandum with justification for a new science of quantum Geophysics in www.vvkuz.ru. By that time a number of works on this subject I had written and published. Since that time, my scientific interest is associated exclusively with this science. Now I published a dozen articles and made some conference reports. My book called "Quantum Geophysics" is conceived. An excellent book al-Khalili and McFadden "Life on the edge. Your first book about quantum biology" [4] was a deciding factor. I used a picture from this book (Fig.5, p. 290) above and I turn to the next picture of this book (Fig. 6).

"The three strata of reality. The top layer is the visible world, filled with objects such as falling apples, cannonballs, steam trains and airplanes, whose motions are described by Newtonian mechanics. Lying beneath is the thermodynamic layer of billiard-ball-like particles whose motion is almost entirely random. This layer is responsible for generating the "order from disorder" laws that govern the behavior of objects such as steam engines. The next layer down is the layer of fundamental particles ruled by orderly quantum laws. The visible features of most of the objects that we see around us appear to be rooted in either the Newtonian or thermodynamic layers but living organisms have roots that penetrate right down to the quantum bedrock of reality [4]."

The earthquake source (Fig. 6) is a chain of HB protons which are in a coherent state. After a decoherence occurs, SW arises and then by the above mentioned script. HB protons of the source can be quantum linked with HB protons of with supercooled water in clouds ("roots of clouds" reach the field of quantum mechanics). Shock crystallization may occur in a cloud as an earthquake aftershock. Reaching ionosphere the resulting SW is converted to SW on the electrons, which is recorded then by GPS methods. Such a cloud is able to generate not only the lightning but also the dark lightning in which water clusters are coupled into a chain so that a voltage of several $\mathrm{MeV}$ instantly appears between the chain ends.

This article deals with some, not all quantum aspects of geophysics. Among the latter, for example, are quantum effects occurring at the boundary of the Earth inner core where a phase change leading to a reverse of geomagnetic field polarity proceeds, etc. [20]. Let us note the role of quantum linked particles which accumulation in atmosphere causes an abrupt climate change, behaving as the flicker-noise. 


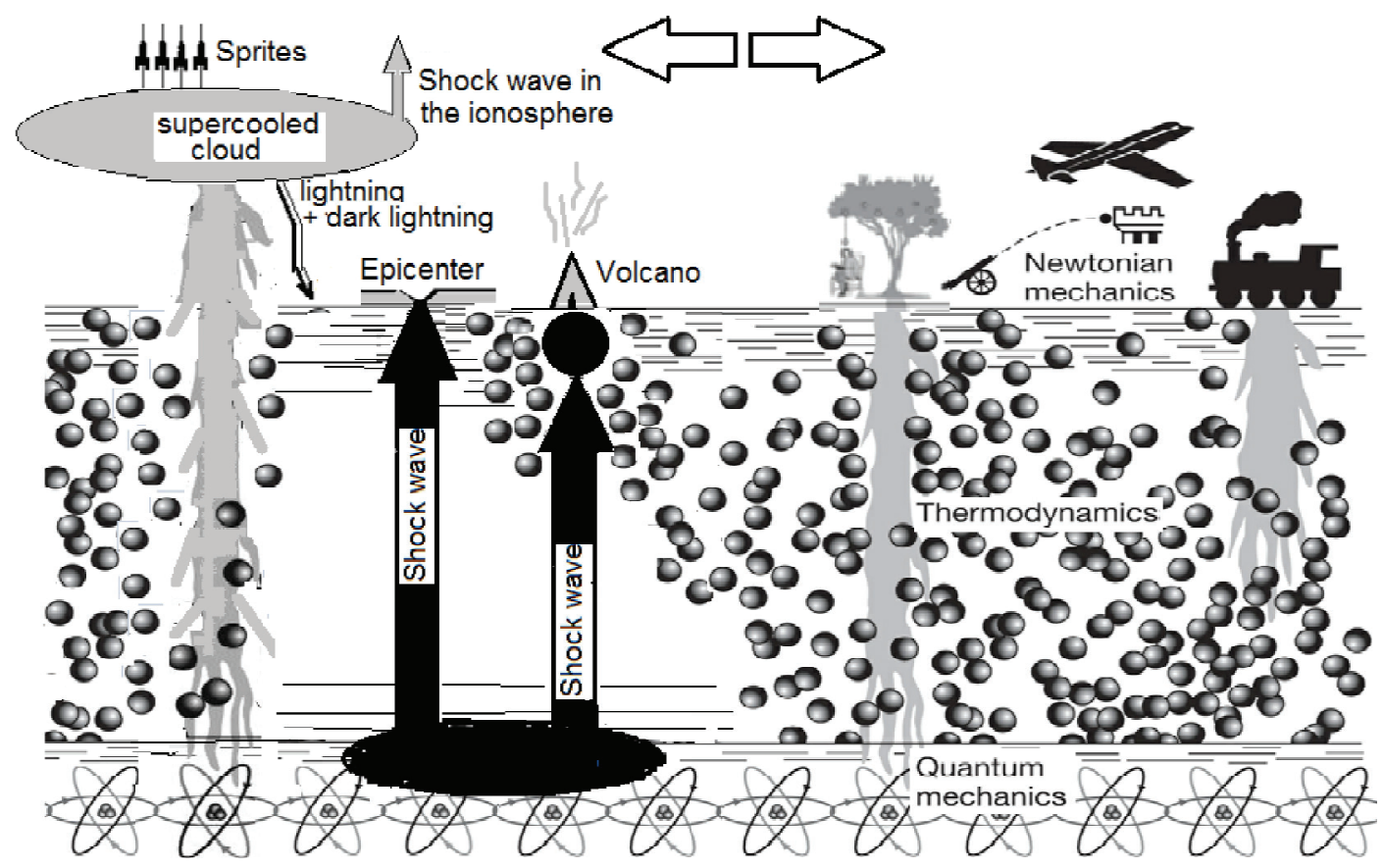

Fig. 6. The three strata of reality. Right part $(\rightarrow)-$ given at [4]. Left part $(\leftarrow)-$ my addition of phenomena and geologic processes discussed here

\section{References}

[1] J. Sonner, Phys. Rev. Lett. 111, 211603 (2013)

[2] R.V. Bunity, S.D.H. Hsu, Phys. Lett. 718(2), 233-236 (2012)

[3] P. Csermely, Weak Links: The universal key to the stability of networks and complex systems (Springer, 2009)

[4] J. Al-Khalili, J. McFadden, Life on the Edge. The coming of quantum biology (Bantam Press, 2014)

[5] S.I. Doronin, Quantum magic (2007), ISBN: 978-5-9573-0844-7

[6] V.D. Shkilev, About the numbers and fractals from the perspective of quantum mechanics, URL:http://www.gramota.net/materials/1/2012/1/21.html (2012)

[7] J. Al-Khalili, Paradox. The nine greatest enigmas in science (Bantam Press, 2012)

[8] V.V. Kuznetsov, Physical mesomechanics 12(6), 87-96 (2009)

[9] K. Kusunose, X. Lei, O. Nishizawa, T. Satoh, PEPI 67(1-2), 194-199 (1991)

[10] S.N. Zhurkov , V.S. Kuksenko, V.A. Petrov, et al., Physical processes in earthquake foci: Sat. scientific (Nauka, Moscow, 1980) 78-85

[11] R.H. Dicke, Phys. Rev. 93, 99 (1954)

[12] R.A. Bertlmann, A. Zeilinger, Aspect A. Bell's theorem: the naïve view of an experimentalist. Quantum [Un] speakables - From Bell to Quantum information (Springer, 2002)

[13] V.V. Kuznetsov, Vestnik KRAUNC. Phys.-Mat. science 3(14), 64-71 (2016) 
[14] D.A. Wiens, J.J. McGuire, P.J.U. Shore, et al., Nature 372, 540-543 (1994)

[15] V.V. Kuznetsov, PMTF 42(4), 184-189 (2001)

[16] L.I. Trakhtenberg, A.A. Fokeev, V.L. Klochikhin, et al., Chemical physics 23(6), 56-68 (2004)

[17] V.V. Kuznetsov, New approaches to the physics of earthquakes and eruptions of volcanoes. Report at the conference. Institute of Volcanology and Seismology. DVO RAN (2015)

[18] V.I. Levitas, R. Ravelo, Proc. Nat. Acad. Sci. USA. 109(33):13204-7 (2012)

[19] V.V. Kuznetsov, Geophysical journal 30(6), 76-92 (2008)

[20] V.V. Kuznetsov, Introduction to the physics of the hot soil (Kamgu, Petropavlovsk-Kamchatsky, 2008) 367

[21] V.V. Kuznetsov, Geology and MCP Siberia, (2017)

[22] V.V. Kuznetsov, Quantum magic 8(1), 1101-1119 (2011)

[23] V.V. Kuznetsov, Volcanology and Seismology (2017)

[24] V.V. Kuznetsov, Vestnik KRAUNC. Phys.-Mat. science 1(10), 34-43 (2015)

[25] N.D. Kuznetsova, V.V. Kuznetsov, Journal of Unconventional Science 3(9), (2015)

[26] V.V. Kuznetsov, Quantum Magic 8(3), 3101-3107 (2011)

[27] V.V. Kuznetsov, Herald kraunts. Phys.-Mat. science 4(15), 63-73 (2016)

[28] V.V. Kuznetsov, Journal of Unconventional Science 11(4), (2016) 